\title{
Dislocation versus grain boundary strengthening in SPD processed metals: non-causal relation between grain size and strength of deformed polycrystals
}

\author{
M.J.Starink \\ Materials Research Group, Faculty of Engineering and the Environment, \\ University of Southampton, Southampton SO17 1BJ, UK
}

\begin{abstract}
:
In metals that are heavily cold deformed, for instance by a severe plastic deformation process, significant strengthening is caused by the high density of defects such as grain boundaries and dislocations. In this work a model for volume-averaged dislocation and grain boundary (GB) creation is used to show that unless significant annihilation of defects post deformation occurs, the dislocation densities and GB densities in the deformed material are closely correlated. The dislocation strengthening effect thus shows a strong correlation with GB strengthening, and correlation of strength or hardness with $d^{1 / 2}$, where $d$ is the grains size, as in a Hall-Petch type plot, can not be taken as an indication that GB strengthening dominates. Instead, in many SPD processed metals and alloys, dislocation strengthening is the dominant strengthening effect, even though a Hall-Petch type plot shows a good linear correlation.
\end{abstract}

Key words: Severe Plastic Deformation (SPD), dislocations, recovery, hardness, grain size

\section{Introduction}

Severe plastic deformation (SPD) has attracted wide attention as a means of improving properties of metals and alloys, and especially improvements in strength have been targeted [1,2,3]. Although grain refinement is often mentioned as being the main factor in strengthening of metals processed by SPD, 
several more detailed analyses which incorporate strengthening models have indicated that dislocation hardening is the main factor determining the strengthening of many SPD processed pure and commercially pure metals $[4,5,6,7,8,9,10,11,12]$, provided SPD and any post-SPD treatment are carried out at temperatures at which recovery is suppressed. Where dislocation density measurements (from diffraction line broadening) are available, this data also tend to indicate that dislocation strengthening is dominant through the well know equation describing the increment of critical resolved shear stress of grains due to dislocations, $\Delta \tau_{\mathrm{d}}[13,14]$ :

$$
\Delta \tau_{d}=\alpha_{1} G b \sqrt{\rho_{i g}}
$$

where $b$ is the Burgers vector, $G$ is the shear modulus, $\rho_{\mathrm{ig}}$ is the (average) dislocation density in the grain, $\alpha_{1}$ is a constant equalling about 0.3 [14].

The aim of this contribution is to analyse the main factors determining dislocation strengthening and grain refinement, aiming to reveal non-causal correlations between grain size and (dislocation) strengthening, which can give rise to misinterpretations of relative strengthening contributions.

\section{Volume averaged model for grain refinement and dislocation generation}

\subsection{General aspects of defect generation}

The quantitative analysis described below uses the volume-averaged dislocation and grain boundary (GB) creation model outlined in $[4,12,15]$. Prior to considering that specific model in Section 2.2 we will first consider the generation of defects (incl. grain boundaries, dislocations and vacancies [16]) from an energetics point of view.

During plastic deformation, mechanical work, $W_{\mathrm{pl}}$ (expressed per unit volume) is imparted to the material and this work, $W_{\mathrm{pl}}$, is converted into the energy of stored defects (incl. grain boundaries, dislocations and vacancies) and heat, $Q$, which will cause a local (relatively small) rise in temperature. We will term the energy stored in grain boundaries as $E_{\mathrm{gb}}(\theta)$, where $\theta$ signifies the GB misorientations angle. The energy stored in in dislocations is $E_{\mathrm{di}}$, the energy stored in twins is $E_{\mathrm{tw}}$, the energy stored in vacancies is $E_{\mathrm{va}}$, and the energy stored at surfaces of (micro) cracks is $E_{\text {cr. }}$ In near adiabatic conditions (i.e. little or no flow of heat/energy through the material during the deformation process) we may then write: 


$$
E_{\mathrm{def}}=E_{\mathrm{gb}}+E_{\mathrm{di}}+E_{\mathrm{tw}}+E_{\mathrm{va}}+E_{\mathrm{cr}}=W_{\mathrm{pl}}-Q
$$

In the treatment below we will apply a number of approximations. It is considered that generally the parameters in SPD processing are chosen such that cracking is avoided, and in these cases $E_{\mathrm{cr}}$ can be ignored. In many materials twinning is limited as compared to other defect generation and $E_{\mathrm{tw}}$ can be ignored. For similar reasons $E_{\mathrm{va}}$ is considered to be negligible. This leaves the main defects to be grain boundaries and dislocations.

New grain boundaries are mostly generated from cell walls (a.k.a. incidental dislocation boundaries (IDBs)), which in turn form by aggregation (or trapping) of dislocations [17]. The GB energy depends on the GB misorientations angle, and to increase the GB misorientation angle dislocations need to be subsumed in the GB [12,15]. This coming together of two defects (in this case a GB and dislocations) thus causes modification of the defect and will in general cause further generation of heat. We can thus consider the process as starting with plastic work, which is converted into heat and defects (initially mostly dislocations), and these defects interact to modify the defects (annihilation of particular defects, clustering of defects to form cell walls, increasing of GB misorientation angles) and generate more heat. As the density of defects increases, so does the interaction between them, and the heat generation increases. This will lead to a stage where the fraction of the work available for generation of defects, $\left(W_{\mathrm{pl}}-Q\right) / W_{\mathrm{pl}}$, decreases to reach the stage where defect densities are stable (and due to interaction with the environment the temperature is stable), and a steady state is reached. From this qualitative consideration of the energetics of the process, we can see that the density of the various types of defects should be correlated, and particularly the density of grain boundaries should be closely correlated to the density of dislocations generated in the course of the deformation process. The densities of both defect types will be closely correlated to the amount of plastic work exerted [18].

2.2 Defect generation in the model for volume-averaged defect and grain boundary creation

To derive the correlation between dislocation and grain boundary densities in a quantitative way we employ the model for the analysis of volume-averaged defect and grain boundary creation described in $[4,12,15]$. This model has proved to be accurate in predicting the grain size and hardness of pure metals $[4,12,15]$, and also correlates well with measured dislocation densities in a range of metals [4]. The model considers that the total cumulative dislocation linelength generated during the deformation processing, 
$L_{\text {gen, }}$ can either be retained in the grains or subsumed in grain boundaries (existing or new ones) $[12,19]$ or annihilated within the grain [20], i.e. [4]:

$$
L_{\mathrm{gb}}+L_{\mathrm{ig}}=\left(1-f_{\mathrm{an}}\right) L_{\mathrm{gen}}
$$

where $f_{\text {an }}$ is the (temperature and material dependent) annihilation fraction [4], $L_{\mathrm{ig}}$ is the total dislocation linelength of dislocations stored in the grain and $L_{\mathrm{gb}}$ is the total dislocation linelength of dislocations that have moved to grain boundaries and have become part of the GB (they are 'subsumed' in the grain boundaries $[12,15])$. To provide a more transparent analysis we will here consider that the volume of the GB with associated dislocations is negligible as compared to the total material volume (i.e. the effective width of grain boundaries is assumed to be negligible). This provides:

$$
\rho_{i g}=\frac{L_{i g}}{V}
$$

where $V$ is the sample volume. We here introduce the factor $A_{1}$ which is the ratio between the $L_{\mathrm{ig}}$ and $L_{\mathrm{gb}}$ :

$$
L_{\mathrm{gb}}=A_{1} L_{\mathrm{ig}}
$$

Little is known about $A_{1}$; it may depend to some limited extend on grain size and it can depend on temperature. $A_{1}$ is thought to be approximately constant for metals deformed to a fixed level of strain at the same temperature.

It has been shown that at the high strain characteristic for SPD, the total dislocation line length that is subsumed in grain boundaries, $L_{\mathrm{gb}}$, is in good approximation proportional to the imposed effective strain, which is in turn inversely proportional to the grain size $[12,15]$. Referring to the analysis in $[12,15]$ it can be seen that the general form of the relation is given by:

$$
\frac{L_{g b}}{V}=A_{2} \frac{\bar{\theta}}{b} \frac{1}{d}
$$

where $d$ is the grain size, $\bar{\theta}$ is average GB misorientation angle and $A_{2}$ is a factor which for the high strains encountered in SPD is in good approximation constant [12,15]. (For further discussion including the effect of average GB misorientation angle on the relation between $L_{\mathrm{gb}}$ and $d$ see $[12,15]$ ). 
Taking the above equations $(1,4,5,6)$ we obtain the following (non-causal) relation between dislocation strengthening and the grain size for a metal that has not undergone post-deformation recovery treatment:

$$
\Delta \tau_{d}=\alpha_{1} G b^{1 / 2} \sqrt{\bar{\theta} \frac{A_{2}}{A_{1}}} d^{-1 / 2}
$$

In the latter equation $\alpha_{1}$ is a constant, $A_{2}$ is expected to be constant, and $A_{1}$ should show little variation with $d . \bar{\theta}$ increases in the course of the deformation, but is expected to reach a stable value that is independent of the type of material $[12,15] . G$ and $b$ are material dependent constants.

The latter equation shows that in polycrystals that have been severely deformed and grain refined by the severe plastic deformation, the strengthening due to dislocations residing in the grain is proportional to $d^{-}$ ${ }^{1 / 2}$. It is stressed that this is even though grain boundaries have no influence on this strengthening: it is a non-causal correlation caused by the fact that the underlying mechanisms for grain refinement and dislocation build-up in grains are closely correlated in terms of the underlying mechanisms. The latter equation provides the same $d^{-1 / 2}$ proportionality suggested by the Hall-Petch relation for grain boundary strengthening $[21,22,23]$ :

$$
\Delta \sigma_{G B}=\frac{k_{H P}}{d_{g}^{1 / 2}}
$$

where $k_{\mathrm{HP}}$ is often referred to as the Hall-Petch constant.

From the above we can see that the common procedure of plotting yield strength (or hardness) as a function $d^{-1 / 2}$ followed by assessment of the correlation / linearity in the plot, will not allow identification of the main strengthening mechanism determining strength / hardness of severely deformed polycrystals. Particularly, a procedure in which $k_{\mathrm{HP}}$ is taken to be a fittable parameter to be determined from the slope of a plot of $\sigma_{\mathrm{y}} \mathrm{vs} d^{-1 / 2}$ will not be able to distinguish whether grain boundaries or dislocations in the grain are responsible for the strengthening; it will merely produce a $k_{\mathrm{HP}}$ value that depends predominantly on $G$ and the dislocation density. Even for one single alloy, a $k_{\mathrm{HP}}$ value determined in this way can vary in a wide range depending on dislocation density. Indeed, it has been pointed out in various publications that reported $k_{\mathrm{HP}}$ values are often inconsistent. 


\section{Experimental data illustrating the correlation}

To test the above points, the correlation of measured grain sizes with measured dislocation sizes was investigated. Using the equations derived from the model for volume averaged dislocation and grain boundary evolution in the previous section we can find the relation between dislocation density and $d$ :

$$
\sqrt{\rho}=b^{-1 / 2} \sqrt{\bar{\theta} \frac{A_{2}}{A_{1}}} d^{-1 / 2}
$$

We first note that it would appear evident that on increasing strain in a single material in the regime where grain size decreases and dislocation densities increase, there will be a correlation between grain size and dislocation density. Such a correlation is for instance seen in data for crystallite sizes and dislocation densities in pure Ta and pure $\mathrm{Nb}$ processed by high pressure torsion (HPT) [6]; Figure 1 plots the measured dislocation densities as a function of measured $\langle\mathrm{x}\rangle^{-1 / 2}$, where $\langle\mathrm{x}\rangle$ is the crystallite size.

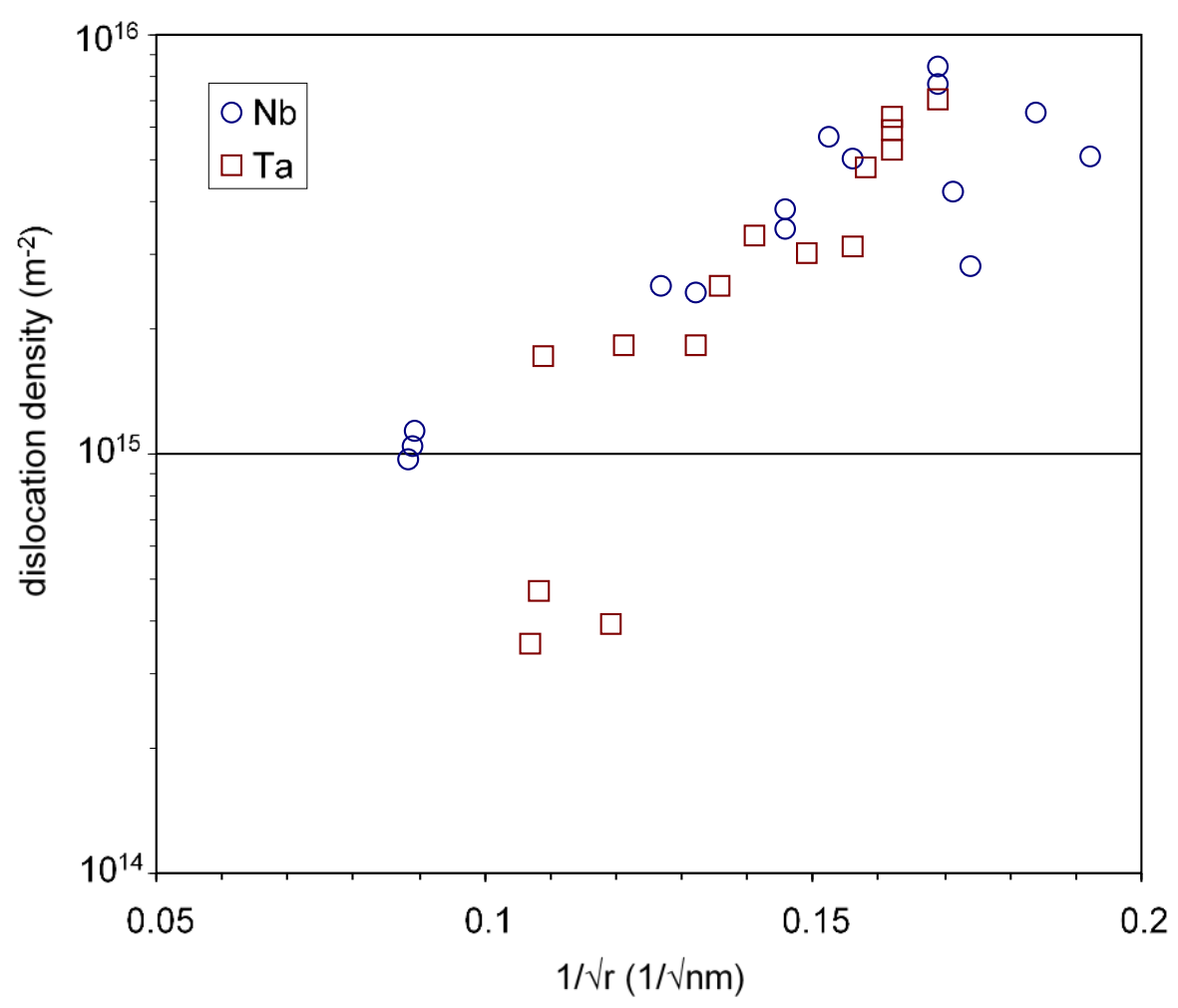

Figure 1 Measured dislocation densities in HPT processed pure Ta and $\mathrm{Nb}$ as a function of measured $\langle\mathrm{x}\rangle^{-1 / 2}$ crystallite size (data from [6]). 
Whilst the latter correlation is evidenced in published work, it is less clear that there would be a correlation between grain size and dislocation density between different metals deformed to high strains (typically 4 to 8) where dislocation densities and grain refinement saturate. To investigate this, data on grain size of pure metals processed by high pressure torsion (HPT) was obtained from the survey in [24]. (Where 2 or more values were reported we here use the average value.) Data on dislocation densities are obtained from the survey in [1] (for original sources see references in [1]), supplemented with data on HPT processed $\mathrm{Ta}$ and $\mathrm{Nb}$ in [6]. For HPT data, HPT processing under a selected pressure in the range $P=1-6 \mathrm{GPa}$ is considered. (Strictly we should consider variation in $b$, but as variation in $b^{-1 / 2}$ is limited we will simplify by not considering differences in $b$.) A plot of dislocation density vs $d^{-1 / 2}$ (Figure 2) shows a strong correlation, indicating that the main point of the present analysis is correct. (It has to be considered here that reported measured dislocation densities of nominally identical deformed metals can sometimes differ by a factor 2 to 3, and hence most of the scatter in Figure 2 is likely to be caused by experimental inaccuracies. Dislocation densities in Figs. 1 and 2 are measured by X-ray diffraction line broadening analysis, see e.g. [25].) A recent analysis Gubicza [16] incorporating solid solution and dispersion strengthened $\mathrm{Al}, \mathrm{Mg}$ and $\mathrm{Cu}$ alloys also shows this correlation.

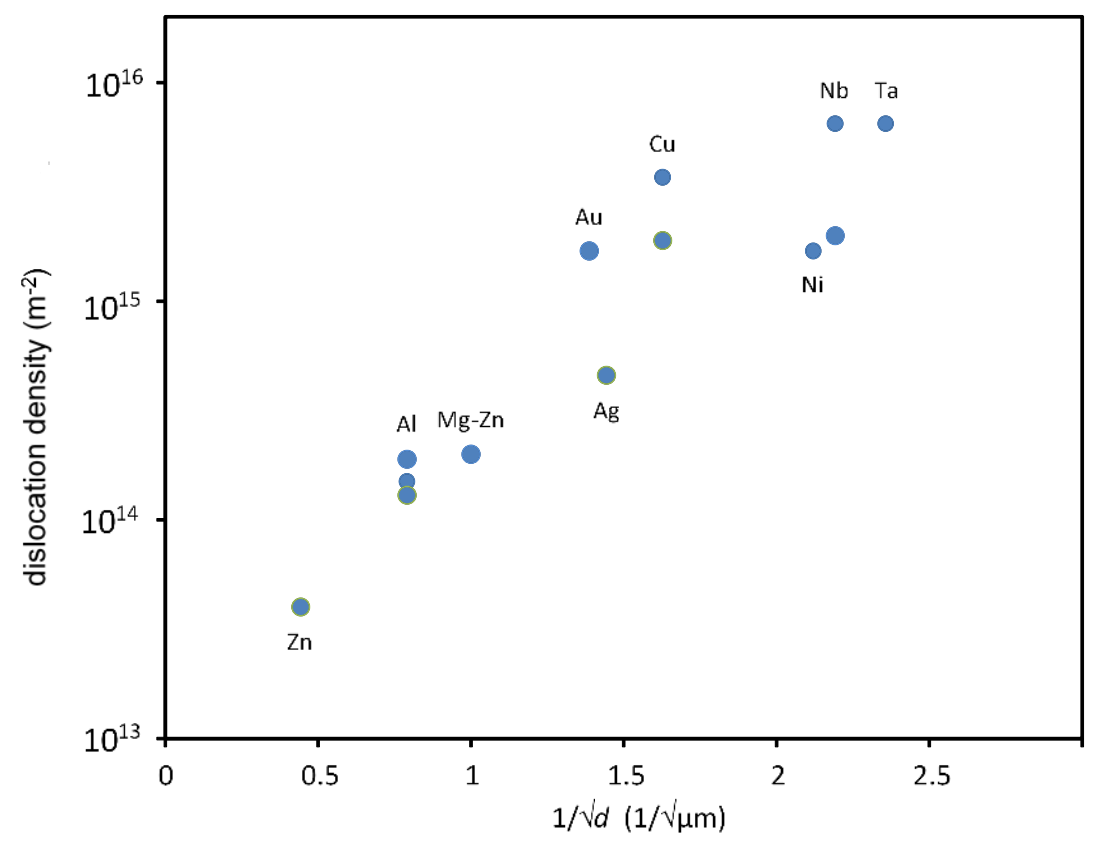

Figure 2 Measured dislocation densities in SPD processed pure metals as a function of measured grain size from HPT processed samples. Data on grain size from [24], data on dislocation densities from the surveys in [1] and [6]. 


\section{Discussion}

The above analysis shows both the model for volume-averaged dislocation and grain boundary (GB) creation as well as experimental data show that in SPD processed materials there is a strong non-causal correlation between $d$ and $\rho_{\mathrm{ig}}$, such that strength will always show a strong positive correlation with $d^{-1 / 2}$ even if GB strengthening is a minor strengthening effect. Also other works have shown a strong correlation between modelled GB strengthening and dislocation strengthening for deformed metals and alloys $[7,18,26,27,28,29]$. This non-causal effect can only be eliminated if a complete recovery treatment is performed, i.e. if all dislocations are annihilated in a recovery heat treatment post SPD. To determine the relative contributions of dislocation strengthening or GB strengthening it is not sufficient to show a correlation with a single microstructural parameter (e.g. $d^{-1 / 2}$ ), and detailed measurements of dislocation density and grain boundaries are needed, combined with modelling. Such work [4-12] clearly shows that for many SPD processed metals and alloys dislocation strengthening is more important than GB strengthening. (For SPD processed pure metals only metals that possess a melting temperature that is less than $\sim 700 \mathrm{~K}$ above the processing temperature show a dislocation strengthening effect that is less than GB strengthening [5].) Thus many works in which strength of SPD processed materials is plotted as a function of $d^{-1 / 2}$ to show a strong correlation need to be considered with caution: unless all dislocations are removed by a recovery treatment such a (linear) correlation is most likely caused by dislocation strengthening.

\section{Conclusions}

A model for volume-averaged dislocation and grain boundary (GB) creation is used to show that unless significant annihilation of defects post deformation occurs, the dislocation densities and grain boundary densities in the deformed metals and alloys are closely correlated. The strengthening due to dislocations will depend linearly on $d^{-1 / 2}$, where $d$ is the grain size. This is similar to the correlation with grain size strengthening suggested by the Hall-Petch type plot. Linearity of a Hall-Petch type plot can not be taken as an indication that GB strengthening dominates. Instead, in many SPD processed metals and alloys, dislocation strengthening is the dominant strengthening effect, even though a Hall-Petch type plot shows a good linear correlation. 


\section{References}

1 R.Z. Valiev, T.G. Langdon, Prog Mater Sci 51, 2006, 881

2 K. Edalati, Z. Horita, Mater Sci Eng A, 652, 2016, 325-352

3 V.M. Segal, Mater Sci Eng A, 97, 1995, 57

4 J. Gubicza, Chapter 7 - Correlation Between Defect Structure and Mechanical Properties of Nanocrystalline Materials, In Defect Structure and Properties of Nanomaterials ( $2^{\text {nd }}$ Edition), Woodhead Publishing, 2017, p 175-223.

5 M.J. Starink, X.Y. Cheng, S.F. Yang, Acta Mater 61, 2012, 183-192

6 B. Jóni, E. Schafler, M. Zehetbauer, G. Tichy, T. Ungár, Acta Mater, 61, 2013, 632-642.

7 Ying Chen, Nong Gao, Gang Sha, S.P. Ringer, M.J. Starink, Acta Mater, 109, 2016, 202-212

8 L. Balogh, T. Ungár, Y. Zhao, Y.T. Zhu, Z. Horita, C. Xu, T.G. Langdon, Acta Mater 56, 2008, 809-820

9 X.G. Qiao, M.J. Starink, N. Gao, Mater Sci Eng A, 513-514, 2009, 52-58

10 J. Gubicza, N.Q. Chinh, J.L. Lábár, S. Dobatkin, Z. Hegedűs, T.G. Langdon, J Alloys Comp 483, 2009, 271 274

11 J. Gubicza, N.Q. Chinh, P. Szommer, A. Vinogradov, T.G. Langdon, Scr Mater 56, 2007, 947-950

12 X.G. Qiao, N. Gao, M.J. Starink, Phil Mag, 92, 2012, 446-470

13 G.I. Taylor, Proc Roy Soc, A145, 1934, p. 362

14 M.F. Ashby, Phil Mag 21, 1970, 399-424

15 M.J. Starink, X.G Qiao, J. Zhang, N. Gao, Acta Mater, 57, 2009, 5796-5811

16 J. Gubicza, Chapter 3 - Defect Structure in Bulk Nanomaterials Processed by Severe Plastic Deformation, In Defect Structure and Properties of Nanomaterials (2nd Edition), Woodhead Publishing, 2017, p. 59-93.

17 M. Cabibbo, Mater Sci Eng A, 560, 2013, 413-432

18 G.S. Dyakonov, S. Mironov, I.P. Semenova, R.Z. Valiev, S.L. Semiatin, Mater Sci Eng A, 701, 2017, $289-$ 301.

19 X.G. Qiao, M.J. Starink, Mater Sci Eng A 531, 2012, 45-50

20 U. Essmann, H. Mughrabi, Phil Mag 40, 1979; 73

21 E.O. Hall, Proc Phys Soc Sect B 64, 1951, 747

22 N.J. Petch, J Iron Steel Inst 26, 1953, 601

23 L.P. Evers, D.M. Parks, W.A.M. Brekelmans, M.G.D. Geers, J Mech Phys Solids, 50, 2002, 2403-2424

24 K. Edalati, Z. Horita, Acta Mater 59, 2011, 6831-6836

25 J. Gubicza, Chapter 2 - Characterization Methods of Lattice Defects, In Defect Structure and Properties of Nanomaterials (2 ${ }^{\text {nd }}$ Edition), Woodhead Publishing, 2017, p 27-57

26 N. Kamikawa, X. Huang, N. Tsuji, N. Hansen, Acta Mater, 57, 2009, 4198-4208.

27 I. Zuiko, R. Kaibyshev, Mater Sci Eng A, 702, 2017, 53-64.

28 S. Malopheyev, V. Kulitskiy, R. Kaibyshev, J All Comp, 698, 2017, 957-966.

29 A.P. Zhilyaev, I. Shakhova, A. Morozova, A. Belyakov, R. Kaibyshev, Mater Sci Eng A, 654, 2016, 131-142. 